\title{
Are Mutations in the DHRS9 Gene Causally Linked to Epilepsy? A Case Report
}

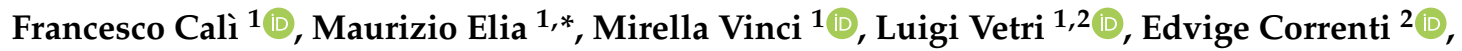 \\ Emanuele Trapolino ${ }^{3}$, Michele Roccella ${ }^{4}{ }^{\circ}$, Francesca Vanadia ${ }^{3}$ and Valentino Romano ${ }^{5}$ \\ 1 Oasi Research Institute-IRCCS, 94018 Troina, Italy; cali@oasi.en.it (F.C.); mvinci@oasi.en.it (M.V.); \\ luigi.vetri@gmail.com (L.V.) \\ 2 Department of Sciences for Health Promotion and Mother and Child Care G. D'Alessandro, \\ University of Palermo, 90127 Palermo, Italy; edvigecorrenti@gmail.com \\ 3 Child Neuropsichiatry Unity, ISMEP-G. di Cristina Hospital-Arnas Civico, 90134 Palermo, Italy; \\ emanuele.trapolino@arnascivico.it (E.T.); francesca.vanadia@arnascivico.it (F.V.) \\ 4 Department of Psychology, Educational Science and Human Movement, University of Palermo, \\ 90128 Palermo, Italy; michele.roccella@unipa.it \\ 5 Department of Biological, Chemical and Pharmaceutical Sciences and Technologies, University of Palermo, \\ 90128 Palermo, Italy; valentino.romano@unipa.it \\ * Correspondence: melia@oasi.en.it; Tel.: +39-0935-936111; Fax: +39-0935-653327
}

Received: 1 July 2020; Accepted: 31 July 2020; Published: 1 August 2020

\begin{abstract}
The DHRS9 gene is involved in several pathways including the synthesis of allopregnanolone from progesterone. Allopregnanolone is a positive modulator of gamma aminobutyric acid (GABA) action and plays a role in the control of neuronal excitability and seizures. Whole-exome sequencing performed on a girl with an early onset epilepsy revealed that she was a compound heterozygote for two novel missense mutations of the DHRS9 gene likely to disrupt protein function. No previous studies have reported the implication of this gene in epilepsy. We discuss a new potential pathogenic mechanism underlying epilepsy in a child, due to a defective progesterone pathway.
\end{abstract}

Keywords: DHRS9; allopregnanolone; GABA; temporal lobe epilepsy; NGS; exome

\section{Introduction}

Genetic studies have revealed a role of the gamma-aminobutyric acid (GABA) system in the pathogenesis of epilepsy and experimental and clinical studies have highlighted the importance of GABA in the treatment of epilepsy [1]. Allopregnanolone (AP) and tetrahydrodeoxycorticosterone (THDOC) are positive allosteric modulators of GABA action at GABAA receptors and play a role in the control of neuronal excitability and seizures $[2,3]$. Allopregnanolone is synthesized in the nervous system from progesterone by the sequential action of two enzymes: $5 \alpha$-reductase type I ( $5 \alpha-$ RI) (SRD5A1 gene), which transforms progesterone into $5 \alpha$-dihydroprogesterone, and $3 \alpha$-hydroxysteroiddehydrogenase ( $3 \alpha$-HSD) (AKR1C2 gene)

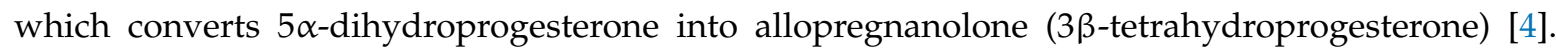
The back-oxidation of $3 \beta$-tetrahydroprogesterone to $5 \alpha$-dihydroprogesterone is catalyzed by a NAD-dependent $3 \alpha$-hydroxysteroid dehydrogenase (DHRS9 gene) [5] (Figure 1). Moreover, Birgit Stoffel-Wagner et al. [6] demonstrated that the 3a-HSD-1 mRNA was not expressed in the hippocampus and temporal lobe of patients with epilepsy. 


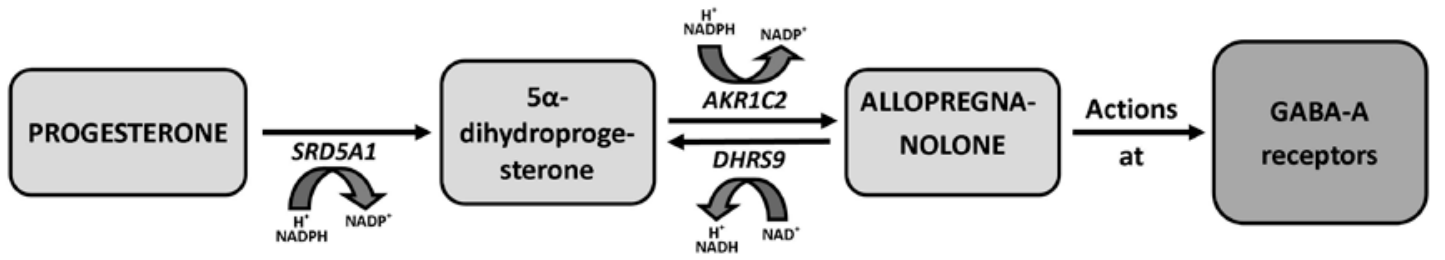

Figure 1. Biosynthetic pathway of the allopregnanolone from progesterone. The patient is a compound heterozygote for two mutations in the DHRS9, preventing the back oxidation of allopregnanolone to 5alpha-dihydroprogesterone (5alpha-DHP) (see text for details and discussion).

\section{Clinical Report}

Here, we describe a 4-year-old girl showing a mild global development delay. The girl had a good adaptation to extrauterine life (APGAR score: 9-10). Her birth weight was $3260 \mathrm{~g}$ (Z-score $-0.36)$, length $50 \mathrm{~cm}$ (Z-score -0.01), and head circumference $35 \mathrm{~cm}$ (Z-score 0.83). Seizures started at two months of life, several times a day, characterized by upward or rightward eye revulsion and immobility; in the following weeks, very frequent epileptic spasms occurred, most frequent on awakening and in falling asleep. Anti-epileptic therapy was administered with valproic acid, levetiracetam, pyridoxine supplementation and adrenocorticotrophic hormone treatment, but seizures were initially drug-resistant. The ictal electroencephalogram showed high-amplitude rhythmic epileptiform discharges at $9 \mathrm{~Hz}$, followed by high-voltage generalized slow waves in the right temporal regions. The interictal EEG was also abnormal with a suppression-burst pattern. The brain magnetic resonance imaging performed at the same age revealed an enlargement of brain cerebrospinal fluid spaces and a thinning of the corpus callosum, without focal lesions. Metabolic investigations resulted normal. Seizures were controlled at 1 year of age, with valproic acid and clonazepam, and EEG did not show epileptiform abnormalities at the last visit, at 4 years of age.

This study was approved by the local Ethics Committee "Comitato Etico IRCCS Sicilia-Oasi Maria SS", Prot. N. 2017/05/31/CE-IRCCS-OASI/9 as of 3 June 2017. The study was conducted in accordance with the Declaration of Helsinki. Written informed consent was obtained from the patient's parents. All investigations were conducted according to the principles expressed in the Declaration of Helsinki. Nuclear DNA from the patient and her parents was isolated from peripheral blood leucocytes. Array-CGH was performed (resolution 400k), but no genomic imbalances were detected. Library preparation and exome enrichment were performed using the Agilent SureSelect V7 (Agilent Technologies, Santa Clara, CA, USA) kit according to the manufacturer's instructions. The quality of post-amplification libraries was assessed using DNA 1000 chips on the BioAnalyzer 2100 (Agilent) and Qubit fluorimetric quantitation using Qubit dsDNA BR Assay Kits (Invitrogen, Carlsbad, CA, USA). An indexed $150 \mathrm{bp}$ paired-end sequencing run was performed on an Illumina HiSeq 3000 instrument at the CRS4 NGS facility. This approach achieved a 75× average coverage over the $36 \mathrm{Mb}$ of genomic regions sequenced, with $95 \%$ regions covered at least $20 \times$. We filtered the identified variants according to: (i) recessive/de novo/X-linked pattern of inheritance, (ii) allele frequencies (Mean Average Frequency, MAF) $<1 \%$ using as reference the following genomic datasets: 1000 Genomes, ESP6500, ExAC, gnomAD.

Exome sequencing performed on nuclear DNA isolated from the patient and her parents showed that she is a compound heterozygote for the c.785C > T (p.Ser262Leu) and c.1036G > C (p.Asp346His) missense mutations of the DHRS9 gene inherited from the mother and the father, respectively.

The two mutations caused changes in two highly conserved amino acid sites in the DHRS9 protein. Furthermore, the "in silico predictive tool" used (Table 1) suggested that the mutations were likely to have a deleterious/damaging effect on DHRS9 function. No other variant was identified in candidate epilepsy genes. 
Table 1. In silico prediction of the effects of missense mutations, c.785C $>\mathrm{T}$ (p.Ser262Leu) and c.1036G > C (p.Asp346His) in the DHRS9 (NM_001289763) gene.

\begin{tabular}{cccc}
\hline In Silico Predictive Tool & \multicolumn{2}{c}{ Prediction/Score } & PHRED-Scaled \\
\hline Mutation & c.785C > T (p.Ser262Leu) & c.1036G > C (p.Asp346His) & \\
\hline dbSNP & rs776765324 & rs11695788 & >30 highly pathogenic; \\
>20 pathogenic
\end{tabular}

* A larger number indicates a higher probability to be damaging.

\section{Discussion}

Based on our findings, we propose that in this patient the epileptic seizures starting in the right temporal regions may be explained by the effect of the missense mutations on DHRS9 function. The absence or dysfunction of DHRS9 could block the back-oxidation of $3 \beta$-tetrahydroprogesterone to $5 \alpha$-dihydroprogesterone (Figure 1). We speculate that in this patient seizures are due to an impairment of allopregnanolone level—due to the above described block—with a consequent effect on inhibitory gabaergic neurons. Stoffel-Wagner et al. [6] have reported the absence of 3a-HSD-1 mRNA in the hippocampus and in the temporal lobe of epileptic patients. Similarly, Lucchi et al. [7] have demonstrated, in their recent study, a significantly reduction in pregnenolone, allopregnanolone and pregnanolone in the hippocampus of epileptic rats. However, interestingly, they found a higher allopregnanolone synthesis in presence of an increased epileptic activity.

Moreover, there is literature evidence that a chronic exposure to increased levels of allopregnanolone (e.g., during stress, menstrual cycle, pregnancy) leads, with an unclear mechanism, to a decrease in the abundance of the GABA-A receptor $\alpha 4$ subunit and a decrease in the expression of the $\alpha 4$ subunit mRNA in the ventral-posteriomedial nucleus of the thalamus [8].

It thus appears that epileptogenesis may result either from an absence of, or a sustained increase in, allopregnanolone. A clear limit of our article is that we have no proof of changes in the brain allopregnanolone concentration. Therefore, more studies are needed on epileptic patients with a defective progesterone pathway, and the use of in vitro functional assays to knock-out the expression of DHRS9 or AKR1C2, such as CRISP-R/Cas 9 and siRNA technologies, will be required to test the validity of the proposed pathogenic model.

Author Contributions: F.C., M.E. designed the whole study; E.C., E.T., M.R., F.V. recruited patient; F.C., M.V. performed whole-exome analysis; F.C., M.V., V.R. analyzed and interpreted the results; F.C., M.E., L.V., V.R. wrote 
the manuscript. All authors approved the submitted version. All authors have read and agreed to the published version of the manuscript.

Funding: This work was partially supported by the Italian Ministry of Health-Ricerca Corrente 2017 and ' 5 per mille' funding.

Acknowledgments: We would like to thank Valeria Chiavetta, Alda Ragalmuto, Angelo Gloria, and Rosanna Galati for their technical contribution.

Conflicts of Interest: Michele Roccella is a Special Issue Editor of Special Issue "New Trends in Neurodevelopmental Disorders". The authors declare that they have no known competing financial interests or personal relationships that could have appeared to influence the work reported in this paper.

\section{References}

1. Treiman, D.M. GABAergic mechanisms in epilepsy. Epilepsia 2001, 42, 8-12. [CrossRef] [PubMed]

2. Beyenburg, S.; Watzka, M.; Clusmann, H.; Blümcke, I.; Bidlingmaier, F.; Elger, C.E.; Stoffel-Wagner, B. Messenger RNA of steroid 21-hydroxylase (CYP21) is expressed in the human hippocampus. Neurosci. Lett. 2001, 308, 111-114. [CrossRef]

3. Galli, R.; Luisi, M.; Pizzanelli, C.; Monteleone, P.; Casarosa, E.; Iudice, A.; Murri, L. Circulating levels of allopregnanolone, an anticonvulsant metabolite of progesterone, in women with partial epilepsy in the postcriticalphase. Epilepsia 2001, 42, 216-219. [PubMed]

4. Melcangi, R.C.; Panzica, G.C. Allopregnanolone: State of the art. Prog. Neurobiol. 2014, 113, 1-5. [CrossRef] [PubMed]

5. Chetyrkin, S.V.; Belyaeva, O.V.; Gough, W.H.; Kedishvili, N.Y. Characterization of a novel type of human microsomal 3alpha -hydroxysteroid dehydrogenase: Unique tissue distribution and catalytic properties. J. Biol. Chem. 2001, 276, 22278-22286. [CrossRef] [PubMed]

6. Stoffel-Wagner, B.; Watzka, M.; Steckelbroeck, S.; Ludwig, M.; Clusmann, H.; Bidlingmaier, F.; Casarosa, E.; Luisi, S.; Elger, C.E.; Beyenburg, S. Allopregnanolone serum levels and expression of 5 alpha-reductase and 3 alpha-hydroxysteroid dehydrogenase isoforms in hippocampal and temporal cortex of patients with epilepsy. Epilepsy Res. 2003, 54, 11-19. [CrossRef]

7. Lucchi, C.; Costa, A.M.; Rustichelli, C.; Biagini, G. Allopregnanolone and pregnanolone are reduced in the hippocampus of epileptic rats, but only allopregnanolone correlates with the seizure frequency. Neuroendocrinology 2020. [CrossRef] [PubMed]

8. Turkmen, S.; Backstrom, T.; Wahlstrom, G.; Andreen, L.; Johansson, I.M. Tolerance to allopregnanolone with focus on the GABA-A receptor. Br. J. Pharmacol. 2011, 162, 311-327. [CrossRef] [PubMed]

(C) 2020 by the authors. Licensee MDPI, Basel, Switzerland. This article is an open access article distributed under the terms and conditions of the Creative Commons Attribution (CC BY) license (http://creativecommons.org/licenses/by/4.0/). 